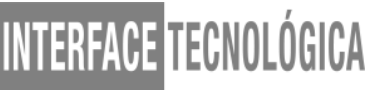

\section{A IMPORTÂNCIA DA ENGENHARIA DE REQUISITOS NO PROCESSO DE DESENVOLVIMENTO DE SISTEMAS DE INFORMAÇÃO}

\section{THE IMPORTANCE OF REQUIREMENTS ENGINEERING IN THE PROCESS OF DEVELOPMENT OF INFORMATION SYSTEMS}

Elvio Carlos da Costa - elvio.costa@fatectq.edu.br Faculdade de Tecnologia de Taquaritinga (FATEC) - SP - Brasil

DOI: 10.31510/infa.v15i1.322

\section{RESUMO}

No atual contexto competitivo, a informação é um grande diferencial, portanto as organizações têm investido pesadamente em sistemas de informação e devem ser totalmente aderentes aos processos de negócio da organização, e essa adesão só é obtido se houver uma correta integração entre usuários e desenvolvedores. O passo fundamental para o sucesso, em qualquer modelo de desenvolvimento de software é a definição e análise de requisitos, pois mesmo se o sistema estiver bem projetado e codificado, porém mal especificado, com certeza, irá causar danos e aborrecimentos para os envolvidos. Nesse sentido, o objetivo desse artigo foi abordar a importância da Engenharia de Requisitos no processo de desenvolvimento de sistemas de informação e definir os processos e atividades oferecidas na Engenharia de Requisitos. A pesquisa foi caracterizada por estudos exploratórios e através de levantamentos de dados (Pesquisa Bibliográfica). Pode-se verificar que a Engenharia de Requisitos depende muito da interação entre os desenvolvedores de sistemas e as pessoas que efetivamente estão envolvidas com o processo de trabalho da organização, minimizando qualquer problema na definição de requisitos por parte do cliente.

Palavras-chave: Sistema de Informação. Engenharia de Requisitos. Desenvolvimento de Software.

\begin{abstract}
In the current competitive context, the information is a big differential. Therefore, the organizations have been investing heavily in systems of information, that should totally be connected to the organization business processes, and this adhesion is only obtained if there is a correct integration between users and developers. The fundamental step for the success, in any model of software development is the definition and analysis of requirements, because even if the system is well projected and codified, however bad specified, it will certainly cause damages and boredom for the people involved. In that sense, the objective of this article is to approach the importance of the Requirements Engineering in the process of development of information systems and to define the processes and activities offered in the Requirements Engineering. The research was characterized by exploratory studies and through surveys of data (Bibliographic Research). It can be seen that Requirements Engineering relies heavily on the interaction between system developers and the people who are effectively involved with the organization's work process, minimizing any problem in the definition of requirements by the customer.
\end{abstract}




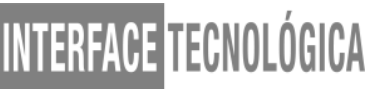

Keywords: Information System. Requirements Engineering. Development of Software.

\section{INTRODUÇÃO}

O desenvolvimento e a implementação de um software visa a melhoria do desempenho organizacional diante dos mercados com a acirrada competição. A indústria de software vem demonstrando crescente interesse em Engenharia de Requisitos e percebendo que o tempo utilizado no entendimento do problema é um excelente investimento.

Segundo Pádua (1998, apud Pressman, 2003) para projetar e construir um software que atenda às necessidades do cliente deve-se entender o que ele realmente deseja. E para tanto, o autor sugere o uso da Engenharia de Requisitos, que inclui o conjunto de tarefas que levam ao entendimento de qual será o impacto do software sobre o negócio, do que o cliente quer e de como os usuários finais vão interagir com o software.

Este artigo tem como objetivo abordar o papel e a importância da Engenharia de Requisitos no desenvolvimento de software e definir os processos e atividades oferecidas na Engenharia de Requisitos.

\section{CONTEXTO E DEFINIÇÕES DE REQUISITOS E ENGENHARIA DE REQUISITOS}

Antes de definir o processo de Engenharia de Requisitos, inicialmente, é fundamental definir o que são requisitos.

Para Alencar (1999) requisito é um conjunto de descrições de como o sistema pretendido deve se comportar, ou um conjunto de propriedades, de atributos do sistema e ou limitações do próprio processo de desenvolvimento do software.

Requisito pode ser definido como "algo que um cliente necessita". Entretanto do ponto de vista do Engenheiro de software, requisitos pode ser definido como "algo que necessita ser projetado". (MACAULAY, 1996, p.72).

Conforme Chichinelli e Cazarini (2003) requisito é uma condição ou uma capacidade que deve ser alcançada ou possuída por um sistema ou componente do sistema, para satisfazer um contrato, um padrão, uma especificação ou outros documentos impostos formalmente. 


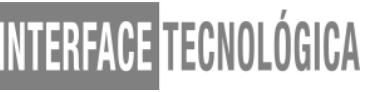

Os requisitos refletem as necessidades dos clientes e usuários de um sistema. Eles devem incluir: justificação para a construção do sistema; o que é pretendido que o sistema faça e quais as restrições de projeto a serem observadas. (KATONYA; SOMMERVILLE, 1995).

Segundo Pádua (1998, apud Pressman, 2003) a Engenharia de Requisitos, como todas as outras atividades da engenharia de software, precisa ser adaptada às necessidades do processo, projeto, produto e do pessoal que está desenvolvendo o trabalho. Na perspectiva do processo de software, a Engenharia de Requisitos começa durante a atividade de comunicação e continua durante a atividade de modelagem, sem ela, o software tem uma alta probabilidade de não satisfazer as necessidades do cliente.

De acordo com Alencar (1996, apud LOUCOPOLOS; KARAKOSTAS, 1999) a Engenharia de Requisitos corresponde ao processo sistemático de desenvolvimento de requisitos, de modo iterativo, cooperativo na análise do problema, com documentação das observações resultantes, com variedade de formatos de representações e checagem da precisão do entendimento obtido.

A Engenharia de Requisitos é uma parte importante no processo de engenharia de software e cada vez mais as organizações investem no aprimoramento das técnicas relacionadas a este assunto.

\subsection{Classificação de Requisitos}

Segundo Turine e Masieiro (1996) os requisitos podem ser classificados como funcionais e não funcionais. Os requisitos funcionais dizem respeito à definição das funções que um sistema ou um componente de sistema deverá fazer, ou seja, as entradas que deverão ser transformada, e as saídas que deverão ser produzidas. São os requisitos que objetivamente cumprem as reais necessidades do usuário do sistema. Enquanto os requisitos não funcionais, dizem respeito às restrições e comportamentos que o software deverá satisfazer. Os requisitos não funcionais ou de qualidade incluem tanto limitações do produto (desempenho, confiabilidade e segurança) como limitações no processo de desenvolvimento (custos, métodos a serem adotados no desenvolvimento e componentes a serem reutilizados). (TURINE; MASIEIRO, 1996). 
De forma geral, a diferença entre requisitos funcionais e não funcionais está no fato dos requisitos funcionais descreverem "o que" o sistema deverá fazer, enquanto os requisitos não funcionais fixam restrições sobre "como" os requisitos funcionais serão implementados.

Segunda Pádua (2003) os requisitos funcionais e não funcionais são derivados dos requisitos organizacionais, que por sua vez são definidos em função do domínio da aplicação. Os requisitos organizacionais dizem respeito às metas da empresa, suas políticas estratégicas adotadas, os empregados da empresa com seus respectivos objetivos, os processos, informações, requisitos não funcionais, enfim representam a estrutura da organização.

\subsection{Funcionalidade, Objetivos e Beneficiados da Engenharia de Requisitos}

Todas as etapas que formam a base de desenvolvimento de software maduro têm sua importância dentro do contexto geral, sempre com o objetivo principal de auxiliar, dentro de suas especificações, o andamento desse desenvolvimento. Com a Engenharia de Requisitos ocorre da mesma forma. Ela possui uma tarefa inicial muito importante nesse processo, pois havendo procedimentos bem definidos que ajudem na tarefa de elicitar os requisitos de um sistema a ser desenvolvido, isso se torna mais fácil e um pouco menos dependente do talento das pessoas e de suas experiências nesse tipo de atividade, evitando boa parte do retrabalho e de inconsistências desses requisitos. (CHICHINELLI; CAZARINI, 2003).

Segundo os mesmos autores, a Engenharia de Requisitos também tem a função de diminuir custos de desenvolvimento através de um processo de amadurecimento de ideias à medida que novos requisitos são expostos, isso se deve a premissa de que quanto mais cedo identificar a mudança menos esforço ela resultará. Isso é feito através, principalmente da conscientização de que os requisitos são mutáveis e através da escolha de um modelo de ciclo de vida adequado. Para fazer o acompanhamento da resolução dos requisitos e da mudança dos mesmos, dando suporte a avaliação de custos e riscos na mudança dos requisitos ao longo da análise ou projeto com a finalidade de avaliar a viabilidade de mudanças dos mesmos no atual instante de desenvolvimento.

De acordo com Chichinelli e Cazarini (2003) um dos principais objetivos da Engenharia de Requisitos é melhorar a modelagem de sistemas e a capacidade de analisá-los, possibilitando maior entendimento de suas características antes da implementação. O papel da Engenharia de Requisitos é também realizar a interação entre requisitantes e desenvolvedores, entre "o que" deve ser feito e "como" deve ser feito. 


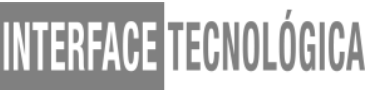

Conforme Cysneiros (2001) os beneficiados com a Engenharia de Requisitos são:

- Os Contratantes: são as pessoas que solicitam os serviços de análise do problema, por terem um documento que assegure a resolução dos problemas referentes aos requisitos funcionais propostos e terem também a garantia da qualidade através de requisitos não funcionais expressados no mesmo documento.

- Os Contratados: são as pessoas que fornecem os serviços de análise de problemas com o documento produzido através da engenharia de software, onde este terá uma visão mais precisa e clara dos verdadeiros requisitos funcionais necessários, não perdendo tempo e sendo mais objetivo, e também podem focalizar a qualidade do produto de acordo com os requisitos não funcionais escolhidos.

- Os futuros Contratados e Contratantes: são as pessoas que podem se beneficiar do reuso da Engenharia de Requisitos através de documentos bem elaborados feitos anteriormente para resolução de problemas semelhantes, diminuindo assim o tempo de desenvolvimento e por conseqüência o custo para o contratante, contextualizando o contratado com o problema a ser resolvido e com as peculiaridades do mesmo em relação a conhecimentos específicos do domínio do problema na área proposta.

\subsection{Processo de Engenharia de Requisitos}

Segundo Kotonya e Sommerville (1998) o processo de Engenharia de Requisitos é um conjunto estruturado de atividades que devem ser seguidas para que se consiga definir, validar e manter um documento de requisitos do sistema. Nas atividades previstas devem constar o levantamento de requisitos, a análise e negociação dos mesmos e a sua validação. O processo ainda deve oferecer diretrizes sobre a programação das atividades, definição de responsabilidades, entradas, saídas de cada uma das atividades, entre outras.

De acordo com os mesmos autores, o processo de Engenharia de Requisitos é muito variável e mostra aos desenvolvedores que não basta apenas buscar informações sobre a funcionalidade dos sistemas, mas é preciso também entender melhor o domínio da aplicação e considerar os aspectos relativos à própria estrutura da organização. No entanto, quatro importantes fatores influenciam o grau de estruturação do processo adotado para Engenharia de Requisitos: maturidade técnica, envolvimento disciplinado, cultura organizacional e o domínio da aplicação. Por melhor que seja um processo, é quase impossível defini-lo como único para todos os casos. 


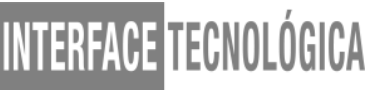

Para Alencar (1996, apud LOUCOPOLOS; KARAKOSTAS, 1999) o processo de Engenharia de Requisitos envolve três atividades fundamentais: Elicitação, Especificação e Validação que correspondem às três preocupações importantes na Engenharia de Requisitos: compreensão, descrição e concordância de um problema. Pode-se notar que independente do processo de Engenharia de Requisitos utilizado, o resultado é um documento de requisitos de software. Este documento é a declaração oficial do que é requerido dos desenvolvedores de sistema.

As quatro atividades mais utilizadas no modelo de processo de Engenharia de Requisitos são:

- Elicitação de Requisitos: é a atividade relacionada à identificação dos requisitos do sistema, a partir de consultas aos representantes de cada grupo de usuários, aos documentos do domínio, ao conhecimento do domínio, e às pesquisas de mercado.

Conforme Pádua(1996, apud JACKSON, 2003) embora a elicitação de requisitos pareça fácil e simples de se obter, a atividade de elicitação de requisitos requer uma análise criteriosa da organização, onde se devem compreender quatro processos:

1) Domínio da Aplicação ou Ambiente: é onde os requisitos particulares dos clientes são encontrados, se não forem identificados corretamente, não se está apto para focalizar os requisitos dos clientes.

2) Problema a Ser Resolvido: o problema no contexto de extração de requisitos é a razão principal para o entendimento, a especialização e o domínio do conhecimento. Este processo é extremamente complexo, pois tem que identificar e determinar o que está errado e consequentemente o que pode ser feito acerca do erro, levando-se em consideração o desejo real do cliente e o que é percebido pelo Engenheiro de Requisitos.

3) Contexto do Negócio: processos organizacionais e fatores políticos geralmente podem influenciar nos requisitos. As dificuldades de comunicação e a falta de padronização no tratamento da informação tornam o processo de validação dos requisitos ainda mais complexo.

4) Informações do Cliente: a fonte de informação cliente é composta por uma variedade de requisitos, expressa de acordo com a posição que a pessoa ocupa na organização e o seu nível de interesse e de comprometimento na identificação de problemas e na procura de solução. 


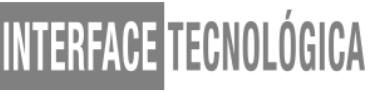

\subsection{Engenharia de Requisitos no processo de desenvolvimento ágil}

O recente surgimento dos métodos ágeis no cenário do desenvolvimento de software pode requerer que a atual área da engenharia de requisitos repense alguns de seus procedimentos. Isto se deve principalmente porque estes métodos abdicam, em parte, de controles e documentação tão presentes nesta área. Os métodos ágeis em geral não mencionam como realizam, por exemplo, a documentação da especificação de requisitos, ou como mantêm a rastreabilidade dos requisitos. Os princípios apresentados pelo manifesto ágil e discutidos por Fowler (2003, p.1) mostram que certos valores relacionados com a área de engenharia de requisitos continuam sendo importantes, como o entendimento dos requisitos, porém preocupam-se em não gerar muita documentação que, justificam, provavelmente nunca será lida.

As discussões em torno da compatibilidade dos métodos ágeis com modelos de qualidade de software, que possuem documentação em todos os processos, principalmente na área de requisitos, têm aumentado significativamente. Turner e Jain (2002, p.161) comentam que, apesar da existência de características distintas entre os métodos ágeis e o modelo CMMI, ambos possuem planos específicos para o desenvolvimento de software e buscam o melhor para que a organização produza software com qualidade.

Segundo Chrissis et al. (2003, p. 18) "o propósito do CMMI é estabelecer um guia para melhorar o processo da organização e sua capacidade para gerenciar o desenvolvimento, aquisição e manutenção de produtos e serviços”. Ahern et al. (2004, p.46) destacam que um dos principais objetivos do modelo CMMI é assegurar a compatibilidade com a norma ISO/IEC 15504 permitindo a análise de áreas independentes do nível de maturidade. O modelo CMMI possui 4 (quatro) disciplinas: Engenharia de Sistemas, Engenharia de Software, Produto Integrado e Desenvolvimento de Processo e, finalmente, a Aquisição. Estas disciplinas, também conhecidas como áreas do conhecimento, auxiliam no planejamento da melhoria do processo de toda organização. A implementação de uma ou mais destas disciplinas ao mesmo tempo com uma única terminologia e infra-estrutura de treinamento e avaliação é considerada uma grande vantagem do modelo CMMI, pois a organização determina em quais disciplinas deseja melhorar seu processo. Estas disciplinas são compostas por áreas de processo que, quando executadas, determinam a melhoria do processo na disciplina escolhida. Segundo (Fiorini, 1998, p.29), área de processo é um conjunto de 


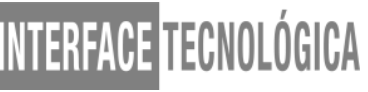

práticas relacionadas em uma área que quando executadas coletivamente satisfazem um conjunto de objetivos importantes para a melhoria significante daquela área.

No que tange ao método ágil Scrum segundo Schwaber (2002, p.2) tem como objetivo definir um processo para projeto e desenvolvimento de software orientado a objeto, que seja focado nas pessoas e que seja indicado para ambientes em que os requisitos surgem e mudam rapidamente. O Scrum também é considerado um método específico para o gerenciamento do processo de desenvolvimento de software. O método baseia-se ainda, conforme Schwaber (2002, p.7), em princípios como: equipes pequenas de, no máximo, 7 pessoas; requisitos que são pouco estáveis ou desconhecidos; e iterações curtas. Divide o desenvolvimento em intervalos de tempos de, no máximo 30 dias, também chamadas de Sprints. Este método não requer ou fornece qualquer técnica ou método específico para a fase de desenvolvimento de software, apenas estabelece conjuntos de regras e práticas gerenciais que devem ser adotadas para o sucesso de um projeto. As práticas gerenciais do Scrum são: Product Backlog, Daily Scrum, Sprint, Sprint Planning Meeting, Sprint Backlog e Sprint Review Meeting. A seguir uma breve descrição do Product Backlog e do Sprint, que são as práticas relacionadas à área de requisitos no método ágil Scrum.

\section{PROCEDIMENTOS METODOLÓGICOS}

Foi realizada uma Pesquisa Bibliográfica, com intuito de coletar informações sobre "A importância da Engenharia de Requisitos no desenvolvimento de Sistemas de Informações", restringindo-se a definirem objetivos e buscar mais informações sobre o tema, bem como publicações e artigos científicos e acadêmicos em torno do assunto em questão.

A pesquisa foi caracterizada por estudos exploratórios e através de levantamentos de dados (Pesquisa Bibliográfica). De acordo com Cervo e Bervian (2002) a Pesquisa Bibliográfica procura explicar um problema a partir de referências teóricas publicadas em documentos, buscando se conhecer e analisar as contribuições culturais ou científicas do passado existente sobre um determinado assunto, tema ou problema, cujos objetivos são: evitar duplicidade de pesquisa, determinar a contribuição para a base do conhecimento e obter fundamentação teórica para o desenvolvimento do projeto. 


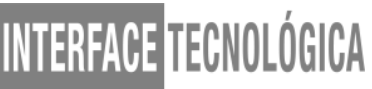

\section{RESULTADOS E DISCUSSÃO}

De acordo com a pesquisa bibliográfica realizada neste estudo, pode-se verificar o quanto a engenharia de requisitos é importante para o desenvolvimento de sistema. Nesse sentido, em suma, a Engenharia de Requisitos traz benefícios a ambas as partes, tanto com diminuição de custos para os contratantes, como em uma melhor contextualização e um maior controle sobre os requisitos para o contratado, e também propõe o reuso dos documentos produzidos, ou seja, incita a reutilização do conhecimento adquirido através da iteração entre as partes ao longo do processo de Engenharia de Requisitos. (CYSNEIROS, 2001).

Destaca-se, também, que os conceitos de produção e gerência de requisitos devem ser considerados em conjunto ao se definir estratégias de trabalho com requisitos nas organizações.

A cada fase do ciclo de vida do software é produzido um documento que contém uma representação distinta do software a ser desenvolvido. Cada um desses documentos representa o software em um determinado nível de abstração. Um dos artefatos inicial do processo de desenvolvimento de software é a sua especificação de requisitos, pois é a base para as demais atividades de desenvolvimento e sua qualidade é fundamental para o sucesso do projeto. (STÁBILE; CAZARINI, 2003)

Para os mesmos autores, uma especificação de requisitos bem elaborada é prérequisito para um software de qualidade, embora não seja garantia disso. Desta forma, durante a produção de requisitos há a necessidade de possuir, além das atividades essenciais de levantamento e especificação, atividades relacionadas à garantia da qualidade. A produção de requisitos baseia-se em quatro atividades:

- Levantamento: relaciona-se à obtenção dos requisitos do software. Para isto, analistas e engenheiros de software trabalham com clientes e usuários finais para descobrir o problema a ser resolvido, os serviços do sistema, o desempenho necessário, restrições de hardware e outras informações. Existem algumas técnicas que apóiam as atividades de levantamento de requisitos, como: entrevistas, prototipação, entre outros.

- Registro: uma vez identificados e negociados, os requisitos devem ser documentados para que possam servir de base para o restante do processo de desenvolvimento. Os requisitos são documentados em um nível apropriado de detalhe. Em geral é produzido um documento de especificação de requisitos, de forma que todos os stakeholders (é o termo utilizado para 
designar todos os envolvidos no projeto, direta ou indiretamente, ou que tenham interesse no resultado do projeto) possam entendê-los. O registro dos requisitos num documento próprio facilita o controle de alterações de todos os envolvidos na manutenção dos requisitos, bem como a geração de versões do documento e a facilidade de acesso por todos os envolvidos.

- Verificação: examina a especificação do software de forma a assegurar que todos os requisitos foram definidos sem ambigüidades, inconsistências ou omissões, detectando e corrigindo possíveis problemas ainda durante a fase de definição dos requisitos. Neste contexto, o custo da correção de defeitos aumenta na medida em que o processo de desenvolvimento progride. Revisão de artefato de software é uma abordagem eficiente e de baixo custo para encontrar defeitos logo após terem sido introduzidos, reduzindo o retrabalho e melhorando a qualidade dos produtos.

- Validação: é a atividade que obtém a aceitação do cliente sob determinado artefato, ou seja, aprovar junto ao cliente os requisitos que foram especificados. Embora aparentemente simples, esta atividade pode ter bastante dificuldade pelo cliente ou mesmo por um processo de validação inadequado utilizado pela empresa.

\section{CONSIDERAÇÕES FINAIS}

A Engenharia de Requisitos define, sem dúvida, um dos mais importantes conjuntos de atividades a serem realizadas em projetos de desenvolvimento de software. Embora não garanta a qualidade dos produtos gerados, é um pré-requisito básico para que se obtenha sucesso no desenvolvimento do sistema de informação.

No processo de Engenharia de Requisitos, não basta apenas buscar informações, mas é necessário também considerar aspectos mais amplos como: objetivos, missão e visão da organização, regras do negócio, política, cultura e estratégia organizacional, entre outros.

É importante salientar que a Engenharia de Requisitos depende muito da interação entre os desenvolvedores de sistemas e as pessoas que efetivamente estão envolvidas com o processo de trabalho da organização, minimizando qualquer problema na definição de requisitos por parte do cliente. Por mais que não se deseje, os requisitos estarão sempre mudando durante o desenvolvimento de um sistema e quão melhor for o processo de Engenharia de Requisitos desenvolvido por todos na empresa, menores serão os problemas encontrados e função de toda a dificuldade que envolve a parte da análise. 


\section{REFERÊNCIAS}

AHERN, Dennis. CLOUSE, Aaron. TURNER, Richard. CMMI Distilled: a practical introduction to integrated process improvement. Boston: Addison Wesley, 2004.

ALENCAR, F. M. R. Mapeando a modelagem organizacional em especificações precisas. Recife, 1999. 304f. Tese (Doutorado em Informática) - Centro de Informática, Universidade Federal de Pernambuco, 1999.

CERVO, Amado L.; BERVIAN, Pedro A. Metodologia Científica. 5.ed. São Paulo: Person Prentice Hall, 2002.

CHICHINELLI, Micheli; CAZARINI, Edson Walmir. Definição dos requisitos: Um dos problemas do processo de desenvolvimento de Sistemas de Informação. In: SIMPÓSIO DE ENGENHARIA DE PRODUÇÃO, 9.,2003. Anais eletrônicos...Bauru: SIMPEP/UNESP, 2003. Disponível em: <http://www.simpep.feb.unesp.br/anais_simpep_aux.php?e=9>. Acesso em: 12.fev.2017.

CHRISSIS, Mary B.; KONRAD M.; SHRUM S. CMMI: Guidelines for Process Integration and Product Improvement. SEI, Addison Wesley, 2003.

CYSNEIROS, Luiz M. Requisitos Não Funcionais: Da Elicitação ao Modelo Conceitual. 2001. 224f. Tese (Doutorado e Ciências da Computação) - Departamento de Informática, Pontifício Universidade Católica do Rio de Janeiro, 2001.

FIORINI, Soeli. STAA, Arndt, BAPTISTA R. Engenharia de software com CMM. Rio de Janeiro: Brasport, 1998.

FOWLER, Martin. The New Methodology. Disponível em: <http://www.martinfowler.com/articles/newMethodology.html>. Acesso em: 12 fev. 2017.

KOTONYA, G.; SOMMERVILLE, I. Requirements Enginnering With Viewpoints. Cooperative System Engineering Group - Technical Report CSEG/10/1995. Computing Department, Lancaster University, 1995.

KOTONYA, G.; SOMMERVILLE, I. Requirements Engineering - Processes and Techniques. Chichester, England : John Wiley \& Sons Inc., 1998.

MACAULAY, L. Requirements Engineering. Berlin: Springer Verlag, 1996.

PÁDUA, Fabiana Serralha Miranda de. A importância da técnica de modelagem organizacional EKD no desenvolvimento de diagramas Use Case. 2003. 130f. Dissertação (Mestrado em Engenharia de Produção) - Universidade de São Paulo, São Carlos, 2003.

STÁBILE, Samuel; CAZARINI, Edson Walmir. O enfoque tecnológico dos Sistemas de Informação. In: SIMPÓSIO DE ENGENHARIA DE PRODUÇÃO, 9.,2003. Anais 
eletrônicos...Bauru: SIMPEP/UNESP, 2003. Disponível em: <http://www.simpep.feb.unesp.br/anais_simpep_aux.php?e=9>. Acesso em: 12.fev.2017.

SCHWABER, Ken; BEEDLE, Mike. Agile Software Development with SCRUM. Prentice Hall, 2002.

TURINE, M. A. S.; MASIERO, P. C. Especificação de Requisitos: Uma introdução. Relatório Técnico. ICMSC:USP, 1996.

TURNER, Richard. JAIN, Apurva. Agile Meets CMMI: Culture clash or common cause. XP/Agile Universe. 2002, p.153-165. 